\title{
PFAFFIAN EQUATIONS SATISFIED BY DIFFERENTIAL MODULAR FORMS
}

\author{
Alexandru Buium
}

\begin{abstract}
The ring of (ordinary) isogeny covariant differential modular forms introduced in [3] was shown in [1] to be described by two basic forms introduced in [3] and [1] respectively. We prove that these two forms satisfy a simple triangular system of Pfaffian equations (in characteristic zero). The equation giving the form in [1] is "integrable by quadratures" which gives a closed form expression for this form; the equation giving the form in [3] is shown not to be "integrable by quadratures".
\end{abstract}

\section{Introduction}

Differential modular forms were introduced in [3]; they are analogues of usual modular forms in a larger geometry [2] obtained from usual algebraic geometry by "adjoining" a "Fermat quotient operation". An aspect of the theory of differential modular forms (which has no analogue in the theory of classical modular forms) is the presence of objects that behave well with respect to isogenies, called isogeny covariant differential modular forms; cf. [3]. As shown in [1] the story of the "ordinary" isogeny covariant forms is essentially told by two fundamental such forms, $f_{\text {jet }}$ and $f^{\partial}$. The form $f_{\text {jet }}$ was introduced in [3]; two equivalent definitions were given for it, one based on crystalline cohomology and the other based on the "arithmetic" Manin map in [2]. The Fourier expansion of $f_{j e t}$ was computed in [3] and the reduction $\bmod p$ of $f_{j e t}$ was computed in [5]. The form $f^{\partial}$ was introduced in [1] via crystalline cohomology and, in that same paper, its Fourier expansion and reduction $\bmod p$ were computed. In spite of the above mentioned results the forms $f_{\text {jet }}$ and $f^{\partial}$ remain quite mysterious and it is reasonable to look for some new, complementary ways of understanding them. This is the motivation of the present paper in which we show that these two forms satisfy a simple, entirely explicit, triangular system of Pfaffian equations. The Pfaffian equation for $f^{\partial}$ can be "integrated by a simple quadrature" hence, as a Corollary, we will be able to give a closed form formula for $f^{\partial}$, up to an "integration constant". Remarkably, once $f^{\partial}$ has been determined, the Pfaffian equation for $f_{\text {jet }}$ has a unique solution; this can be interpreted as saying, in

Received February 13, 2004.

The author was supported in part by NSF Grant \#0096946.

Key words and phrases. modular forms, Serre operator. 
particular, that the Pfaffian equation for $f_{j e t}$ is not "integrable by quadratures" in any reasonable way. So, in some sense, the form $f_{\text {jet }}$ is more elusive than $f^{\partial}$.

Here is the main idea of the paper. Our forms $f_{j e t}$ and $f^{\partial}$ are, as we shall recall below, power series in 4 variables. In order to compute the differential $d f$ of either $f=f_{j e t}$ or $f=f^{\partial}$ we need to know the effect on $f$ of 4 linearly independent vector fields. The paper [1] provides 2 independent vector fields together with their effect on $f$. These vector fields are "differential analogues" of the classical Serre and Euler operators. One needs then two more vector fields to complete the picture and this is what we are going to provide in this paper. The 2 new vector fields to be introduced here are, in some sense "conjugates" of the vector fields in [1]. Here the word "conjugate" is understood in an arithmetic sense but also in a sense similar to that of conjugate variables in mechanics. The latter analogy comes via the analogy between the arithmetic jet spaces in [2] and usual jet spaces (phase spaces) in differential geometry.

It is worth noting that our characteristic zero results here have a characteristic $p$ counterpart; cf. [4]. The characteristic $p$ story is easier to deal with (the 4 vector fields mentioned above being replaced in [4] by the classical Serre and Euler operators) but on the other hand the characteristic $p$ differential equation in [4] has a flavour (and consequences) of its own.

Our note is organized as follows. In Section 2 we review the definition of $f_{\text {jet }}$ and $f^{\partial}$ and some of their properties, following [3] and [1], and we state our main results. In Section 3 we introduce our main tool, the conjugate operators. In Section 4 we conclude the proof of our main results.

\section{Main concepts and results}

Let us review some basic definitions from [3]. Start with indeterminates $a_{4}, a_{6}$, set $\Delta:=-2^{6} a_{4}^{3}-2^{4} 3^{3} a_{6}^{2}$ and consider the ring

$$
M^{0}:=\mathbf{Z}_{p}\left[a_{4}, a_{6}, \Delta^{-1}\right]^{\wedge}
$$

where $\mathbf{Z}_{p}$ is the ring of $p$-adic integers $(p \geq 5)$ and the superscript ${ }^{\wedge}$ means " $p$-adic completion".

Now let $a_{4}^{\prime}, a_{6}^{\prime}$ be new indeterminates and consider the ring

$$
M^{1}:=M^{0}\left[a_{4}^{\prime}, a_{6}^{\prime}\right]^{\wedge}=\mathbf{Z}_{p}\left[a_{4}, a_{6}, a_{4}^{\prime}, a_{6}^{\prime}, \Delta^{-1}\right]^{\wedge}
$$

The elements of this ring will be referred to as $\delta$-modular functions. For any such element $f$ we usually write $f=f\left(a_{4}, a_{6}, a_{4}^{\prime}, a_{6}^{\prime}\right)$; note that it makes sense to substitute the four variables in $f$ by any elements in a $p$-adically complete ring in which the value of $\Delta$ is invertible. Let $\phi: M^{0} \rightarrow M^{1}$ be the unique $\mathbf{Z}_{p}$-algebra homomorphism such that $\phi\left(a_{4}\right)=a_{4}^{p}+p a_{4}^{\prime}, \phi\left(a_{6}\right)=a_{6}^{p}+p a_{6}^{\prime}$, write $f^{\phi}$ instead of $\phi(f)$, and define $\delta: M^{0} \rightarrow M^{1}$ by the formula $\delta f=\left(f^{\phi}-f^{p}\right) / p$.

Let $\Lambda, \Lambda^{\prime}$ be two more variables and denote by

$$
\phi: M^{0}\left[\Lambda, \Lambda^{-1}\right]^{\wedge} \rightarrow M^{1}\left[\Lambda, \Lambda^{\prime}, \Lambda^{-1}\right]^{\wedge}
$$


the unique $\mathbf{Z}_{p}$-algebra homomorphism lifting $\phi: M^{0} \rightarrow M^{1}$ and satisfying $\phi(\Lambda)=\Lambda^{p}+p \Lambda^{\prime}$. We continue to write $f^{\phi}:=\phi(f)$ for any $f \in M^{0}\left[\Lambda, \Lambda^{-1}\right]^{\wedge}$. Also we consider the map

$$
\delta: M^{0}\left[\Lambda, \Lambda^{-1}\right]^{\wedge} \rightarrow M^{1}\left[\Lambda, \Lambda^{\prime}, \Lambda^{-1}\right]^{\wedge}, \quad \delta f:=\frac{f^{\phi}-f^{p}}{p} .
$$

For any expression of the form $w=n_{0}+n_{1} \phi \in \mathbf{Z} \oplus \mathbf{Z} \phi$ we set

$$
f^{w}=f^{n_{0}} \cdot\left(f^{\phi}\right)^{n_{1}} \text { for } f \in M^{0}\left[\Lambda, \Lambda^{-1}\right]^{\wedge} .
$$

For any $\delta$-modular function $f \in M^{1}$ write

$$
f * \Lambda:=f\left(\Lambda^{4} a_{4}, \Lambda^{6} a_{6}, \delta\left(\Lambda^{4} a_{4}\right), \delta\left(\Lambda^{6} a_{6}\right)\right),
$$

$f * \Lambda \in M^{1}\left[\Lambda, \Lambda^{\prime}, \Lambda^{-1}\right]^{\wedge}$. Say that $f$ is a $\delta$-modular form of weight $w \in \mathbf{Z} \oplus \mathbf{Z} \phi$ if

$$
f * \Lambda=\Lambda^{w} \cdot f .
$$

More generally assume one is given a $\delta$-modular form $g \not \equiv 0 \bmod p$ (of some weight) and an element

$$
f \in M^{1}\left[g^{-1}\right]^{\wedge}
$$

one says that $f$ has weight $w$ if Equation 7 holds.

Similarly if $q$ and $q^{\prime}$ are variables consider the $\operatorname{ring} \mathbf{Z}_{p}((q))^{\wedge}:=\mathbf{Z}_{p}[[q]]\left[q^{-1}\right]^{\wedge}$, the ring homomorphism

$$
\phi: \mathbf{Z}_{p}((q))^{\wedge} \rightarrow \mathbf{Z}_{p}((q))\left[q^{\prime}\right]^{\wedge}, F \mapsto \phi(F)=F^{\phi}:=F\left(q^{p}+p q^{\prime}\right),
$$

and the map

$$
\delta: \mathbf{Z}_{p}((q))^{\wedge} \rightarrow \mathbf{Z}_{p}((q))\left[q^{\prime}\right]^{\wedge}, F \mapsto \delta F:=\frac{F^{\phi}-F^{p}}{p} .
$$

Following [3] we define then the $\left(q, q^{\prime}\right)-$ Fourier expansion map

$$
\begin{gathered}
M^{1} \rightarrow \mathbf{Z}_{p}((q))\left[q^{\prime}\right]^{\wedge} \\
f \mapsto f\left(q, q^{\prime}\right):=f\left(a_{4}(q), a_{6}(q), \delta\left(a_{4}(q)\right), \delta\left(a_{6}(q)\right)\right),
\end{gathered}
$$

where

$$
\begin{gathered}
a_{4}(q):=-2^{-4} 3^{-1} E_{4}(q), a_{6}(q):=-2^{-5} 3^{-3} E_{6}(q), \\
E_{2 m}(q):=1-\frac{4 m}{B_{2 m}} \sum_{n=1}^{\infty} \sigma_{2 m-1}(n) q^{n},
\end{gathered}
$$

the usual normalized Eisenstein series, with $B_{2 m}$ the Bernoulli numbers. More generally, for a $\delta$-modular function $g \not \equiv 0 \bmod p$ we have an induced $\left(q, q^{\prime}\right)-$ Fourier expansion map

$$
M^{1}\left[g^{-1}\right]^{\wedge} \rightarrow\left(\mathbf{Z}_{p}((q))\left[q^{\prime}\right]^{\wedge}\left[g\left(q, q^{\prime}\right)^{-1}\right]\right)^{\wedge} .
$$

We have the following $\left(q, q^{\prime}\right)$-expansion principle; cf. [3], Proposition 7.21 plus the Remark following that Proposition. 
Proposition 2.1. [3] For any weight $w \in \mathbf{Z} \oplus \mathbf{Z} \phi$ the $\left(q, q^{\prime}\right)-$ Fourier expansion map 10 restricted to the set of elements of weight $w$ is injective.

By the way recall from [7] that $E_{p-1}(q)$ and $E_{p+1}(q)$ belong to $\mathbf{Z}_{p}((q))$ and are images of unique forms

$$
E_{p-1}, E_{p+1} \in \mathbf{Z}_{p}\left[a_{4}, a_{6}\right]
$$

of weight $p-1$ and $p+1$ respectively. Also recall from [6] that $E_{2}(q)$ belongs to $\mathbf{Z}_{p}((q))$ and is the image of a unique form

$$
P \in \mathbf{Z}_{p}\left[a_{4}, a_{6}, E_{p-1}^{-1}\right]^{\wedge}
$$

of weight 2 which we can refer to as the Ramanujan form. The reduction mod $p, \bar{P}$, of $P$ is given as follows. Cf. [1], Lemma 3.1.

Lemma 2.2. [1] $\bar{E}_{p+1}=\bar{P} \cdot \bar{E}_{p-1}$ in $\mathbf{F}_{p}\left[a_{4}, a_{6}, \bar{E}_{p-1}^{-1}\right]$.

The following was proved in [3], Corollary 7.26.

Theorem 2.3. [3] There exists a unique $\delta$-modular form $f_{\text {jet }} \in M^{1}$ of weight $-1-\phi$ with $\left(q, q^{\prime}\right)-$ Fourier expansion

$$
f_{\text {jet }}\left(q, q^{\prime}\right)=\frac{1}{p} \log \frac{q^{\phi}}{q^{p}}=\sum_{n=1}^{\infty}(-1)^{n-1} p^{n-1} n^{-1}\left(\frac{q^{\prime}}{q^{p}}\right)^{n} .
$$

Uniqueness follows, of course, from Proposition 2.1.

The following result follows from [1] by putting together Theorem 5.1, Proposition 5.2, and Equation 5.1 in loc. cit.

Theorem 2.4. [1] There exist unique elements $f^{\partial}, f_{\partial} \in M^{1}\left[E_{p-1}^{-1}\right]^{\wedge}, f^{\partial} f_{\partial}=1$, of weights $\phi-1$ and $1-\phi$ respectively, with $\left(q, q^{\prime}\right)-$ Fourier expansions

$$
f^{\partial}\left(q, q^{\prime}\right)=f_{\partial}\left(q, q^{\prime}\right)=1 \text {. }
$$

Their reductions mod $p$ are given by

$$
\bar{f}^{\partial}=\bar{E}_{p-1}, \bar{f}_{\partial}=\bar{E}_{p-1}^{-1} \in \mathbf{F}_{p}\left[a_{4}, a_{6}, a_{4}^{\prime}, a_{6}^{\prime}, \bar{\Delta}^{-1}, \bar{E}_{p-1}^{-1}\right] .
$$

Uniqueness follows, of course, from Proposition 2.1.

Finally recall from [7] that we have at our disposal the classical Serre operator

$$
\partial=-2^{3} 3^{2} a_{6} \frac{\partial}{\partial a_{4}}+2^{4} a_{4}^{2} \frac{\partial}{\partial a_{6}}: \mathbf{Z}_{p}\left[a_{4}, a_{6}\right] \rightarrow \mathbf{Z}_{p}\left[a_{4}, a_{6}\right] .
$$

Note that $\partial \Delta=0$. If $\bar{\partial}: \mathbf{F}_{p}\left[a_{4}, a_{6}\right] \rightarrow \mathbf{F}_{p}\left[a_{4}, a_{6}\right]$ is the reduction $\bmod p$ of $\partial$ then

$$
\bar{\partial} \bar{E}_{p-1}=\bar{E}_{p+1}
$$

cf. [7], p. 165. By Equation 13 and Lemma 2.2 we may consider the following element which will play a role later:

$$
\frac{1}{p}\left(P-\frac{\partial E_{p-1}}{E_{p-1}}\right) \in \mathbf{Z}_{p}\left[a_{4}, a_{6}, E_{p-1}^{-1}\right]^{\wedge}
$$


In what follows set

$$
S^{0}=M^{0}\left[E_{p-1}^{-1}\right]^{\wedge}, \quad S^{1}=M^{1}\left[E_{p-1}^{-1}\right]^{\wedge} .
$$

We denote by $\Omega_{S^{1}}^{1}$ the $p$-adic completion of the module of Kahler differentials of $S^{1} / \mathbf{Z}_{p}$; then $\Omega_{S^{1}}^{1}$ is a free $S^{1}$-module with basis with basis

$$
d a_{4}, d a_{6}, d a_{4}^{\prime}, d a_{6}^{\prime} .
$$

Similarly we define $\Omega_{S^{0}}^{1}$; it is a free $S^{0}$-module with basis with basis $d a_{4}, d a_{6}$. Denote by $\Omega_{S^{1}}^{i}$ the $i$-th wedge product of $\Omega_{S^{1}}^{1}$; we may then consider the de Rham complex

$$
S^{1} \stackrel{d}{\rightarrow} \Omega_{S^{1}}^{1} \stackrel{d}{\rightarrow} \Omega_{S^{1}}^{2} \stackrel{d}{\rightarrow} \ldots
$$

and similarly for $S^{0}$. The map $\phi: S^{0} \rightarrow S^{1}$ induces maps $\phi^{*}: \Omega_{S^{0}}^{i} \rightarrow \Omega_{S^{1}}^{i}$ which commute with the differentials $d$. Consider the following 1-forms:

$$
\begin{gathered}
\omega_{0}=\frac{6 a_{6} d a_{4}-4 a_{4} d a_{6}}{\Delta} \in \Omega_{S^{0}}^{1}, \\
\eta_{0}=\frac{d \Delta}{12 \Delta} \in \Omega_{S^{0}}^{1}, \\
\omega_{1}=p^{-1} \phi^{*} \omega_{0}=p^{-1} \cdot \frac{6 a_{6}^{\phi} d\left(a_{4}^{\phi}\right)-4 a_{4}^{\phi} d\left(a_{6}^{\phi}\right)}{\Delta^{\phi}} \in \Omega_{S^{1}}^{1}, \\
\eta_{1}=p^{-1} \phi^{*} \eta_{0}=p^{-1} \frac{d\left(\Delta^{\phi}\right)}{12 \Delta^{\phi}} \in \Omega_{S^{1}}^{1} .
\end{gathered}
$$

Then $P \omega_{0}$ and $\eta_{0}$ are trivially checked to be closed (i.e. in the kernel of $d$ ). Therefore $\nu_{0}:=P \omega_{0}-\eta_{0}$ and $\phi^{*} \nu_{0}=p P^{\phi} \omega_{1}+p \eta_{1}$ are also closed. Here is the first main result of our note:

Theorem 2.5. The pair $(X, Y)=\left(f^{\partial}, f_{\text {jet }}\right) \in\left(S^{1}\right)^{\times} \times S^{1}$ satisfies the following system of equations in $\Omega_{S^{1}}^{1}$ :

$$
\begin{gathered}
d X=X \cdot\left(\nu_{0}-\phi^{*} \nu_{0}\right), \\
d Y=Y \cdot\left(\nu_{0}+\phi^{*} \nu_{0}\right)-12 X^{-1} \omega_{0}+12 X \omega_{1} .
\end{gathered}
$$

The system of Equations 17 and 18 should be viewed as a triangular Pfaffian system satisfied by $\left(f^{\partial}, f_{j e t}\right)$. As it is, this system is not linear because of the presence of the $X^{-1}$ term in Equation 18; but, of course, one can rewrite the system as a linear system by noting that $X^{*}:=X^{-1}$ satisfies the equation

$$
d X^{*}=X^{*} \cdot\left(-\nu_{0}+\phi^{*} \nu_{0}\right) \text {. }
$$

It turns out that Equation 17 is "solvable by quadratures" which will help us give a closed form formula for $f^{\partial}$. We say that a form $\omega \in \Omega_{S^{1}}^{1}$ is exact if $\omega=d g$ for some $g \in S^{1}$; such a $g$ is referred to as a primitive of $\omega$ and we write $g=\int \omega$. Note that closed forms need not be exact. Then we will deduce, from Theorem 2.5, the following: 
Corollary 2.6. The form

$$
\omega:=\frac{1}{p}\left(P-\frac{\partial E_{p-1}}{E_{p-1}}\right) \omega_{0}-\eta_{0}-P^{\phi} \omega_{1}+\eta_{1} \in \Omega_{S^{1}}^{1}
$$

is exact and there exists a primitive $\int \omega$ such that

$$
f^{\partial}=E_{p-1} \cdot \exp \left(p \int \omega\right)
$$

In the above statement $\exp : p S^{1} \rightarrow 1+p S^{1}$ is the usual $p$-adic exponential function. The next result looks at the space of all solutions to the Equations 17 and 18:

Theorem 2.7. 1) If $X_{1}, X_{2} \in S^{1}$ are any two non-zero solutions of Equation 17 , not in $p S^{1}$, then $X_{2}=\lambda X_{1}$ for some $\lambda \in \mathbf{Z}_{p}^{\times}$.

2) If $\left(X, Y_{1}\right)$ and $\left(X, Y_{2}\right)$ are two solutions, in $\left(S^{1}\right)^{\times} \times S^{1}$, of the system of Equations 17 and 18 then $Y_{1}=Y_{2}$. Equivalenty, the Equation

$$
d Z=Z \cdot\left(\nu_{0}+\phi^{*} \nu_{0}\right)
$$

has no non-zero solution in $S^{1}$.

Corollary 2.8. The element $Y=f_{\text {jet }}$ is the unique solution in $S^{1}$ of the equation

$$
d Y=Y \cdot\left(\nu_{0}+\phi^{*} \nu_{0}\right)-12 f_{\partial} \omega_{0}+12 f^{\partial} \omega_{1} .
$$

In particular Equation 21 is not "integrable by quadratures" in any reasonable sense; indeed, any definition of "integrability by quadratures" should allow the presence of an "integration constant" in the "general solution".

\section{The conjugate operators}

In addition to the Serre operator in Equation 12 one can consider the Euler operator

$$
\mathcal{D}=4 a_{4} \frac{\partial}{\partial a_{4}}+6 a_{6} \frac{\partial}{\partial a_{6}}: \mathbf{Z}_{p}\left[a_{4}, a_{6}\right] \rightarrow \mathbf{Z}_{p}\left[a_{4}, a_{6}\right] .
$$

The following operator, called $\delta$-Serre operator, played a key role in [1]:

$$
\partial_{1}:=-2^{3} 3^{2} a_{6}^{\phi} \frac{\partial}{\partial a_{4}^{\prime}}+2^{4} a_{4}^{2 \phi} \frac{\partial}{\partial a_{6}^{\prime}}: M^{1} \rightarrow M^{1}
$$

Also the following operator (which we can refer to as the $\delta$-Euler operator) was considered in [1]

$$
\mathcal{D}_{1}:=4 a_{4}^{\phi} \frac{\partial f}{\partial a_{4}^{\prime}}+6 a_{6}^{\phi} \frac{\partial f}{\partial a_{6}^{\prime}}: M^{1} \rightarrow M^{1} .
$$


The main idea of the present paper is to introduce the following conjugate $\delta$-Serre operator

$$
\partial_{0}:=-2^{3} 3^{2} a_{6}\left(\frac{\partial}{\partial a_{4}}-a_{4}^{p-1} \frac{\partial}{\partial a_{4}^{\prime}}\right)+2^{4} a_{4}^{2}\left(\frac{\partial}{\partial a_{6}}-a_{6}^{p-1} \frac{\partial}{\partial a_{6}^{\prime}}\right): M^{1} \rightarrow M^{1},
$$

and also the following conjugate $\delta$-Euler operator

$$
\mathcal{D}_{0}:=4\left(a_{4} \frac{\partial f}{\partial a_{4}}-a_{4}^{p} \frac{\partial f}{\partial a_{4}^{\prime}}\right)+6\left(a_{6} \frac{\partial f}{\partial a_{6}}-a_{6}^{p} \frac{\partial f}{\partial a_{6}^{\prime}}\right): M^{1} \rightarrow M^{1} .
$$

Remark 3.1. The operator $\partial_{0}$ can be characterized as the unique $\mathbf{Z}_{p}$-derivation lifting the classical Serre operator $\partial$ in Equation 12 and satisfying

$$
\partial_{0}\left(f^{\phi}\right)=0
$$

for all $f \in M^{0}$. A similar description holds for $\mathcal{D}_{0}$.

Recall the following easy facts from [1], p. 251.

Lemma 3.2. [1] If $f \in M^{1}\left[g^{-1}\right]^{\wedge}$ has weight $w$ then

$$
\frac{\partial f}{\partial a_{4}^{\prime}} \text { and } \frac{\partial f}{\partial a_{6}^{\prime}}
$$

have weights $w-4 \phi$ and $w-6 \phi$ respectively. In particular $\partial_{1} f$ has weight $w+2 \phi$.

Lemma 3.3. [1] If $f \in M^{1}\left[g^{-1}\right]^{\wedge}$ has weight $w=n_{0}+n_{1} \phi$ then

$$
\mathcal{D}_{1} f=p n_{1} f .
$$

The above two lemmas have analogues for the conjugate operator as follows.

Lemma 3.4. If $f \in M^{1}\left[g^{-1}\right]^{\wedge}$ has weight $w$ then

$$
\frac{\partial f}{\partial a_{4}}-a_{4}^{p-1} \frac{\partial f}{\partial a_{4}^{\prime}} \text { and } \frac{\partial f}{\partial a_{6}}-a_{6}^{p-1} \frac{\partial f}{\partial a_{6}^{\prime}}
$$

have weights $w-4$ and $w-6$ respectively. In particular $\partial_{0} f$ has weight $w+2$.

Proof. First note that

$$
\frac{\partial}{\partial a_{4}}\left[\delta\left(\Lambda^{4} a_{4}\right)\right]=a_{4}^{p-1}\left(\Lambda^{4 \phi}-\Lambda^{4 p}\right) .
$$

Taking $\partial / \partial a_{4}$ in Equation 7 we get

$$
\begin{gathered}
\Lambda^{w} \frac{\partial f}{\partial a_{4}}=\left(\frac{\partial f}{\partial a_{4}} * \Lambda\right) \cdot \Lambda^{4}+\left(\frac{\partial f}{\partial a_{4}^{\prime}} * \Lambda\right) \cdot \frac{\partial}{\partial a_{4}}\left[\delta\left(\Lambda^{4} a_{4}\right)\right] \\
=\Lambda^{4} \cdot\left[\left(\frac{\partial f}{\partial a_{4}}-a_{4}^{p-1} \frac{\partial f}{\partial a_{4}^{\prime}}\right) * \Lambda\right]+\Lambda^{4 \phi} a_{4}^{p-1} \cdot\left(\frac{\partial f}{\partial a_{4}^{\prime}} * \Lambda\right), \quad \text { by Equation } 29 \\
=\Lambda^{4} \cdot\left[\left(\frac{\partial f}{\partial a_{4}}-a_{4}^{p-1} \frac{\partial f}{\partial a_{4}^{\prime}}\right) * \Lambda\right]+a_{4}^{p-1} \Lambda^{w} \frac{\partial f}{\partial a_{4}^{\prime}}, \quad \text { by Lemma } 3.2 ;
\end{gathered}
$$

this proves the assertion for $a_{4}$. The assertion for $a_{6}$ is proved similarly. 
Lemma 3.5. If $f \in M^{1}\left[g^{-1}\right]^{\wedge}$ has weight $w=n_{0}+n_{1} \phi$ then

$$
\mathcal{D}_{0} f=n_{0} f \text {. }
$$

Proof. Note that

$$
\begin{gathered}
\left(\frac{\partial \Lambda^{w}}{\partial \Lambda}\right)_{\mid \Lambda=1, \Lambda^{\prime}=0}=n_{0}+p n_{1}, \\
\left(\frac{\partial}{\partial \Lambda}\left(\delta\left(\Lambda^{4} a_{4}\right)\right)\right)_{\mid \Lambda=1, \Lambda^{\prime}=0}=4 p a_{4}^{\prime}, \\
\left(\frac{\partial}{\partial \Lambda}\left(\delta\left(\Lambda^{6} a_{6}\right)\right)\right)_{\mid \Lambda=1, \Lambda^{\prime}=0}=6 p a_{6}^{\prime} .
\end{gathered}
$$

Taking $\partial / \partial \Lambda$ in Equation 7 , setting $\Lambda=1, \Lambda^{\prime}=0$, and using Equations 30, 31, and 32 we get

$$
4\left(a_{4} \frac{\partial f}{\partial a_{4}}+p a_{4}^{\prime} \frac{\partial f}{\partial a_{4}^{\prime}}\right)+6\left(a_{6} \frac{\partial f}{\partial a_{6}}+p a_{6}^{\prime} \frac{\partial f}{\partial a_{6}^{\prime}}\right)=\left(n_{0}+p n_{1}\right) f .
$$

We conclude by subtracting Equation 28 from Equation 33.

As in [1], where the Fourier expansion of $\partial_{1} f$ was computed for $f$ a $\delta$-modular form, we need a formula for $\partial_{0} f$. Consider the following derivation

$$
\theta_{0}:=q \frac{\partial}{\partial q}-q^{p} \frac{\partial}{\partial q^{\prime}}: \mathbf{Z}_{p}((q))\left[q^{\prime}\right]^{\wedge} \rightarrow \mathbf{Z}_{p}((q))\left[q^{\prime}\right]^{\wedge}
$$

$\theta_{0}$ can be characterized as being the unique derivation lifting

$$
\theta:=q \frac{\partial}{\partial q}: \mathbf{Z}_{p}((q))^{\wedge} \rightarrow \mathbf{Z}_{p}((q))^{\wedge}
$$

and satisfying

$$
\theta_{0}\left(F^{\phi}\right)=0
$$

for all $F \in \mathbf{Z}_{p}((q))^{\wedge}$. In particular

$$
\theta_{0} \delta F=-F^{p-1} \theta F
$$

for all $F \in \mathbf{Z}_{p}((q))^{\wedge}$. One should view $\theta_{0}$ as the "conjugate" of the operator

$$
\theta_{1}:=q^{\phi} \frac{\partial}{\partial q^{\prime}}
$$

considered in [1].

With $P$ the Ramanujan form, cf. Equation 11, the following was proved in [1]:

Lemma 3.6. [1] If $f \in M^{1}\left[g^{-1}\right]^{\wedge}$ has weight $w=n_{0}+n_{1} \phi$ then

$$
12 \theta_{1}\left(f\left(q, q^{\prime}\right)\right)=\left(\partial_{1} f+p n_{1} P^{\phi} f\right)\left(q, q^{\prime}\right) .
$$

We need a "conjugate analogue" of the above Lemma: 
Lemma 3.7. If $f \in M^{1}\left[g^{-1}\right]^{\wedge}$ has weight $w=n_{0}+n_{1} \phi$ then

$$
12 \theta_{0}\left(f\left(q, q^{\prime}\right)\right)=\left(\partial_{0} f+n_{0} P f\right)\left(q, q^{\prime}\right) .
$$

Proof. Using Equation 36 we get

$$
\begin{aligned}
& \theta_{0}\left(\delta\left(a_{4}(q)\right)\right)=-a_{4}^{p-1} \cdot \theta\left(a_{4}(q)\right), \\
& \theta_{0}\left(\delta\left(a_{6}(q)\right)\right)=-a_{6}^{p-1} \cdot \theta\left(a_{6}(q)\right) .
\end{aligned}
$$

On the other hand recall from [7], p. 161, that

$$
\begin{aligned}
& 12 \theta\left(a_{4}(q)\right)=\left(4 P a_{4}+\partial a_{4}\right)(q), \\
& 12 \theta\left(a_{6}(q)\right)=\left(6 P a_{6}+\partial a_{6}\right)(q) .
\end{aligned}
$$

We now have the following computation using Equations 39, 40, 41, 42, and Lemma 3.5:

$$
\begin{gathered}
12 \theta_{0}\left(f\left(q, q^{\prime}\right)\right)=12 \frac{\partial f}{\partial a_{4}}\left(q, q^{\prime}\right) \cdot \theta\left(a_{4}(q)\right)+12 \frac{\partial f}{\partial a_{6}}\left(q, q^{\prime}\right) \cdot \theta\left(a_{6}(q)\right) \\
+12 \frac{\partial f}{\partial a_{4}^{\prime}}\left(q, q^{\prime}\right) \cdot \theta_{0}\left(\delta\left(a_{4}(q)\right)\right)+12 \frac{\partial f}{\partial a_{6}^{\prime}}\left(q, q^{\prime}\right) \cdot \theta_{0}\left(\delta\left(a_{6}(q)\right)\right) \\
=\left[\partial_{0} f+P \cdot\left(4\left(a_{4} \frac{\partial f}{\partial a_{4}}-a_{4}^{p} \frac{\partial f}{\partial a_{4}^{\prime}}\right)+6\left(a_{6} \frac{\partial f}{\partial a_{6}}-a_{6}^{p} \frac{\partial f}{\partial a_{6}^{\prime}}\right)\right)\right]\left(q, q^{\prime}\right) \\
=\left(\partial_{0} f+n_{0} P f\right)\left(q, q^{\prime}\right) .
\end{gathered}
$$

Recall from [1] the following:

Proposition 3.8. [1] The following equalities hold in $S^{1}$ :

$$
\begin{gathered}
\partial_{1} f_{j e t}=12 f^{\partial}+p P^{\phi} f_{j e t}, \\
\partial_{1} f^{\partial}=-p P^{\phi} f^{\partial} .
\end{gathered}
$$

We need a "conjugate analogue" of the above Proposition:

Proposition 3.9. The following equalities hold in $S^{1}$ :

$$
\begin{gathered}
\partial_{0} f_{j e t}=-12 f_{\partial}+P f_{j e t}, \\
\partial_{0} f^{\partial}=P f^{\partial} .
\end{gathered}
$$


Proof. In order to check the first equation note that both its members have weight $1-\phi$. By the $\left(q, q^{\prime}\right)$ - expansion principle, cf. Proposition 2.1, it is enough to check that both members have the same Fourier $\left(q, q^{\prime}\right)$-expansion. Note that

$$
\begin{gathered}
\theta_{0}\left(f_{\text {jet }}\left(q, q^{\prime}\right)\right)=\theta_{0}\left(\frac{1}{p} \log \frac{q^{\phi}}{q^{p}}\right), \quad \text { by Theorem } 2.3 \\
=\frac{1}{p} \theta_{0}\left(\frac{q^{\phi}}{q^{p}}\right) \cdot \frac{q^{p}}{q^{\phi}}=-1, \quad \text { by Equation } 35 .
\end{gathered}
$$

On the other hand one has the following computation for the Fourier $\left(q, q^{\prime}\right)$ expansions:

$$
\begin{aligned}
\left(\partial_{0} f_{j e t}\right)\left(q, q^{\prime}\right) & =12 \theta_{0}\left(f_{j e t}\left(q, q^{\prime}\right)\right)+\left(P f_{j e t}\right)\left(q, q^{\prime}\right), \quad \text { by Lemma } 3.7 \\
= & -12+\left(P f_{j e t}\right)\left(q, q^{\prime}\right), \quad \text { by Equation } 43 \\
= & \left(-12 f_{\partial}+P f_{j e t}\right)\left(q, q^{\prime}\right), \quad \text { by Theorem } 2.4 .
\end{aligned}
$$

This concludes the proof of the first equation. The proof of the second equation is entirely similar.

The above two Propositions have analogues involving the Euler operators that follow directly from Lemmas 3.3 and 3.5:

Corollary 3.10. The following equalities hold in $S^{1}$ :

$$
\begin{aligned}
& \mathcal{D}_{1} f^{\partial}=p f^{\partial}, \quad \mathcal{D}_{1} f_{j e t}=-p f_{\text {jet }}, \\
& \mathcal{D}_{0} f^{\partial}=-f^{\partial}, \quad \mathcal{D}_{0} f_{\text {jet }}=-f_{\text {jet }} .
\end{aligned}
$$

\section{Proof of the main results}

The dual, $\Theta_{S^{1}}$, of $\Omega_{S^{1}}^{1}$ has basis

$$
\frac{\partial}{\partial a_{4}}, \frac{\partial}{\partial a_{6}}, \frac{\partial}{\partial a_{4}^{\prime}}, \frac{\partial}{\partial a_{6}^{\prime}} .
$$

Similarly the dual, $\Theta_{S^{0}}$, of $\Omega_{S^{0}}$ has a basis consisting of the first 2 components of the above basis. Note that

$$
\left(\begin{array}{c}
\partial_{0} \\
\mathcal{D}_{0} \\
\partial_{1} \\
\mathcal{D}_{1}
\end{array}\right)=A\left(\begin{array}{c}
\partial / \partial a_{4} \\
\partial / \partial a_{6} \\
\partial / \partial a_{4}^{\prime} \\
\partial / \partial a_{6}^{\prime}
\end{array}\right)
$$

where

$$
\begin{gathered}
A=\left(\begin{array}{cc}
A_{11} & A_{12} \\
0 & A_{22}
\end{array}\right), \\
A_{11}=\left(\begin{array}{cc}
-2^{3} 3^{2} a_{6} & 2^{4} a_{4}^{2} \\
4 a_{4} & 6 a_{6}
\end{array}\right), \quad A_{12}=\left(\begin{array}{cc}
2^{3} 3^{2} a_{4}^{p-1} a_{6} & -2^{4} a_{4}^{2} a_{6}^{p-1} \\
-4 a_{4}^{p} & -6 a_{6}^{p}
\end{array}\right),
\end{gathered}
$$


and $A_{22}=A_{11}^{\phi}$. Then $\operatorname{det} A=\Delta^{\phi+1}$ so $A$ is invertible hence $\partial_{0}, \mathcal{D}_{0}, \partial_{1}, \mathcal{D}_{1}$ form a basis of $\Theta_{S^{1}}$; the inverse $B=A^{-1}$ is trivially found to be:

$$
B=\left(\begin{array}{cc}
B_{11} & B_{12} \\
0 & B_{22}
\end{array}\right),
$$

where

$$
B_{11}=\frac{1}{\Delta}\left(\begin{array}{cc}
6 a_{6} & -2^{4} a_{4}^{2} \\
-4 a_{4} & -2^{3} 3^{2} a_{6}
\end{array}\right), \quad B_{12}=\frac{1}{\Delta^{\phi}}\left(\begin{array}{cc}
6 a_{4}^{p-1} a_{6}^{\phi} & -2^{4} a_{4}^{2 \phi+p-1} \\
-4 a_{4}^{\phi} a_{6}^{p-1} & -2^{3} 3^{2} a_{6}^{\phi+p-1}
\end{array}\right),
$$

and $B_{22}=B_{11}^{\phi}$. A straightforward computation gives

$$
\left(d a_{4}, d a_{6}, d a_{4}^{\prime}, d a_{6}^{\prime}\right) B=\left(\omega_{0}, \eta_{0}, \omega_{1}, \eta_{1}\right),
$$

where $\omega_{0}, \eta_{0}, \omega_{1}, \eta_{1}$ are as in Equation 16. With the preparation above it is trivial to conclude the proof of Theorem 2.5:

Proof. Using Equation 44 plus Propositions 3.8 and 3.9 and Corollary 3.10 we have

$$
\begin{gathered}
d f^{\partial}=\left(d a_{4}, d a_{6}, d a_{4}^{\prime}, d a_{6}^{\prime}\right) B A\left(\begin{array}{c}
\partial / \partial a_{4} \\
\partial / \partial a_{6} \\
\partial / \partial a_{4}^{\prime} \\
\partial / \partial a_{6}^{\prime}
\end{array}\right) f^{\partial}=\left(\omega_{0}, \eta_{0}, \omega_{1}, \eta_{1}\right) \cdot\left(\begin{array}{c}
\partial_{0} \\
\mathcal{D}_{0} \\
\partial_{1} \\
\mathcal{D}_{1}
\end{array}\right) f^{\partial} \\
=f^{\partial}\left(P \omega_{0}-\eta_{0}-p P^{\phi} \omega_{1}+p \eta_{1}\right) .
\end{gathered}
$$

Similarly

$$
\begin{aligned}
d f_{j e t}=\left(\omega_{0}, \eta_{0}, \omega_{1}, \eta_{1}\right) & \cdot\left(\begin{array}{c}
\partial_{0} \\
\mathcal{D}_{0} \\
\partial_{1} \\
\mathcal{D}_{1}
\end{array}\right) f_{j e t} \\
& =f_{j e t}\left(P \omega_{0}-\eta_{0}+p P^{\phi} \omega_{1}-p \eta_{1}\right)-12 f_{\partial} \omega_{0}+12 f^{\partial} \omega_{1} .
\end{aligned}
$$

Then one can deduce Corollary 2.6 as follows:

Proof. Start by noting that

$$
\begin{gathered}
d E_{p-1}=\left(d a_{4}, d a_{6}\right) B_{11} A_{11}\left(\begin{array}{c}
\partial / \partial a_{4} \\
\partial / \partial a_{6}
\end{array}\right) E_{p-1}=\left(\omega_{0}, \eta_{0}\right)\left(\begin{array}{c}
\partial \\
\mathcal{D}
\end{array}\right) E_{p-1} \\
=E_{p-1}\left(\frac{\partial E_{p-1}}{E_{p-1}} \omega_{0}+(p-1) \eta_{0}\right) .
\end{gathered}
$$




\section{Hence}

$$
\begin{aligned}
d\left(\frac{f^{\partial}}{E_{p-1}}\right) & =E_{p-1}^{-2}\left(E_{p-1} d f^{\partial}-f^{\partial} d E_{p-1}\right) \\
& =\frac{f^{\partial}}{E_{p-1}}\left(P \omega_{0}-\eta_{0}-p P^{\phi} \omega_{1}+p \eta_{1}\right)-\frac{f^{\partial}}{E_{p-1}}\left(\frac{\partial E_{p-1}}{E_{p-1}} \omega_{0}+(p-1) \eta_{0}\right) \\
& =\frac{f^{\partial}}{E_{p-1}} \cdot p \cdot \omega .
\end{aligned}
$$

On the other hand, by Theorem 2.4, we have

$$
\frac{f^{\partial}}{E_{p-1}} \in 1+p S^{1} .
$$

Let $\log : 1+p S^{1} \rightarrow p S^{1}$ be the $p$-adic logarithm and set

$$
U:=\frac{1}{p} \log \frac{f^{\partial}}{E_{p-1}} \in S^{1} .
$$

Then $d U=\omega$ and we are done.

To prove Theorem 2.7 we need the following:

Lemma 4.1. Let $g \in \mathbf{Z}_{p}\left[x_{1}, \ldots, x_{n}\right]^{\wedge}, g \not \equiv 0 \bmod p, h \in \mathbf{Z}_{p}\left[x_{1}, \ldots, x_{n}, g^{-1}\right]^{\wedge}$, $h \not \equiv 0 \bmod p$, and let

$$
f \in\left(\mathbf{Z}_{p}\left[x_{1}, \ldots, x_{n}, g^{-1}\right]^{\wedge}\right)\left[h^{-1}\right]
$$

be such that

$$
\frac{\partial f}{\partial x_{1}}=\ldots=\frac{\partial f}{\partial x_{n}}=0
$$

Then $f \in \mathbf{Z}_{p}$.

Proof. First we prove the assertion of the Lemma for $h=1$. We may replace $\mathbf{Z}_{p}$ by $R:=\hat{\mathbf{Z}}_{p}^{u r}$, the completion of the maximum unramified extension of $\mathbf{Z}_{p}$. Since the residue field of $R$ is infinite there exists $a_{1}, \ldots, a_{n} \in R$ such that $g\left(a_{1}, \ldots, a_{n}\right) \not \equiv$ $0 \bmod p$. Since $\partial / \partial x_{i}$ are invariant under the $R$-automorphism $x_{i} \mapsto x_{i}-a_{i}$ of $R\left[x_{1}, \ldots, x_{n}\right]^{\wedge}$, we may assume $a_{1}=\ldots=a_{n}=0$. Then we have an injection

$$
R\left[x_{1}, \ldots, x_{n}, g^{-1}\right]^{\wedge} \rightarrow R\left[\left[x_{1}, \ldots, x_{n}\right]\right]
$$

and we are done by the (obvious) fact that the only elements in $R\left[\left[x_{1}, \ldots, x_{n}\right]\right]$ killed by all $\partial / \partial x_{i}$ are in $R$. This proves the case $h=1$ of the Lemma. For arbitrary $h$ we take $h_{0} \in \mathbf{Z}_{p}\left[x_{1}, \ldots, x_{n}, g^{-1}\right]$ congruent $\bmod p$ with $h$ in the latter ring and write $h_{0}=h_{1} / g^{N}, h_{1} \in \mathbf{Z}_{p}\left[x_{1}, \ldots, x_{n}\right]$. It is trivial to check that

$$
\left(\left(\mathbf{Z}_{p}\left[x_{1}, \ldots, x_{n}, g^{-1}\right]^{\wedge}\right)\left[h^{-1}\right]\right)^{\wedge} \simeq \mathbf{Z}_{p}\left[x_{1}, \ldots, x_{n},\left(h_{1} g\right)^{-1}\right]^{\wedge}
$$

By the $h=1$ case of our proof, the image of $f$ in $\left(\left(\mathbf{Z}_{p}\left[x_{1}, \ldots, x_{n}, g^{-1}\right]^{\wedge}\right)\left[h^{-1}\right]\right)^{\wedge}$ lies in $\mathbf{Z}_{p}$. But now, the homomorphism from $\left(\mathbf{Z}_{p}\left[x_{1}, \ldots, x_{n}, g^{-1}\right]^{\wedge}\right)\left[h^{-1}\right]$ to its completion is injective (because any ring of fractions of a $p$-adically separated ring in which $p$ is prime and not a zero divisor is again $p$-adically separated, 
provided $p$ does not divide any of the elements of the multiplicative system). We conclude that $f \in \mathbf{Z}_{p}$.

Proof. (of Theorem 2.7) If $X_{1}, X_{2} \in S^{1}$ are as in assertion 1 then $X_{2} d X_{1}=$ $X_{1} d X_{2}$ so $X_{2} / X_{1} \in S^{1}\left[X_{1}^{-1}\right]$ is killed by both $\partial / \partial a_{4}$ and $\partial / \partial a_{6}$. By Lemma 4.1 $X_{2} / X_{1}$ is in $\mathbf{Z}_{p}$ hence in $\mathbf{Z}_{p}^{\times}$.

Now assume $0 \neq Z \in S^{1}$ is a solution to Equation 20 and let us derive a contradiction. Upon combining Equations 17 and 20 we get the following equalities

$$
\begin{gathered}
d(X Z)=X Z \cdot 2 \nu_{0}, \\
d(Z / X)=(Z / X) \cdot 2 \phi^{*} \nu_{0}
\end{gathered}
$$

in $\Omega_{S^{1}}^{1}$. Equation 45 shows that

$$
\frac{\partial}{\partial a_{4}^{\prime}}(X Z)=\frac{\partial}{\partial a_{6}^{\prime}}(X Z)=0
$$

which immediately shows that $V:=X Z \in S^{0}$. Since $\phi^{*}$ and $d$ commute, Equation 45 implies

$$
d\left(V^{\phi}\right)=V^{\phi} \cdot 2 \phi^{*} \nu_{0}
$$

From Equations 47 and 46 we get

$$
\left(V / X^{2}\right) \cdot d\left(V^{\phi}\right)=V^{\phi} \cdot d\left(V / X^{2}\right)
$$

in $\Omega_{S^{1}}$. Since $V \neq 0$ we can write $V=p^{n} W$ with $W \in S^{0} \backslash p S^{0}$ and an equality as in Equation 48 holds with $V$ replaced by $W$. We get that $X^{2} W^{\phi} / W \in S^{1}\left[W^{-1}\right]$ is killed by

$$
\frac{\partial}{\partial a_{4}}, \frac{\partial}{\partial a_{6}}, \frac{\partial}{\partial a_{4}^{\prime}}, \frac{\partial}{\partial a_{6}^{\prime}} .
$$

By Lemma 4.1 we get that $X^{2} W^{\phi} / W$ is in $\mathbf{Z}_{p}$, hence in $\mathbf{Z}_{p}^{\times}$. Since $S^{1}$ is an integral domain we get

$$
X^{2} W^{\phi}=\lambda_{1} W
$$

in $S^{1}$, with $\lambda_{1} \in \mathbf{Z}_{p}^{\times}$. By assertion 1 in the Theorem we have

$$
X=\lambda_{2} f^{\partial},
$$

with $\lambda_{2} \in \mathbf{Z}_{p}^{\times}$. Combining Equations 49 and 50, reducing $\bmod p$, and using Theorem 2.4 we get an equality of the form

$$
\bar{E}_{p-1}^{2} \bar{W}^{p-1}=\bar{\lambda}_{1} \bar{\lambda}_{2}^{-2}
$$

in $S^{1} \otimes \mathbf{F}_{p}$ hence in

$$
S^{0} \otimes \mathbf{F}_{p}=\mathbf{F}_{p}\left[a_{4}, a_{6}, \bar{\Delta}^{-1}, \bar{E}_{p-1}^{-1}\right] .
$$

So there exists a constant $c \in \mathbf{F}_{p}^{\times}$such that

$$
\bar{E}_{p-1} \bar{W}^{\frac{p-1}{2}}=c .
$$


Recall from [7], p. 167, that $\bar{E}_{p-1}$ has no multiple irreducible factors. Also recall that $\bar{\Delta}$ is irreducible in $\mathbf{F}_{p}\left[a_{4}, a_{6}\right]$ and does not divide $\bar{E}_{p-1}$ (as one can see by looking at Fourier expansions). Write $\bar{E}_{p-1}=\bar{G}_{1} \ldots \bar{G}_{s}$ with $\bar{G}_{i}$ irreducible polynomials in $\mathbf{F}_{p}\left[a_{4}, a_{6}\right]$ and write $\bar{W}$ as an irreducible fraction

$$
\bar{W}=\frac{\bar{F}}{\bar{\Delta}^{N} \bar{G}_{1}^{n_{1}} \ldots \bar{G}_{s}^{n_{s}}}
$$

with $n_{i} \geq 0, N \geq 0, \bar{F} \in \mathbf{F}_{p}\left[a_{4}, a_{6}\right]$. Then one can write Equation 52 as

$$
\bar{G}_{1} \ldots \bar{G}_{s} \bar{F}^{\frac{p-1}{2}}=c \bar{\Delta}^{N}\left(\bar{G}_{1}^{n_{1}} \ldots \bar{G}_{s}^{n_{s}}\right)^{\frac{p-1}{2}} .
$$

If all $n_{i}=0$ then we must have $N \geq 1$ but then $\bar{\Delta}$ divides the left hand side of Equation 53, a contradiction. If there is an $i$ such that $n_{i} \geq 1$ then, since $p \geq 5$, we get that $\bar{G}_{i}$ divides

$$
\bar{G}_{1 \ldots} \bar{G}_{i-1} \bar{G}_{i+1} \ldots \bar{G}_{s} \bar{F}^{\frac{p-1}{2}}
$$

which is again a contradiction. This concludes our proof.

\section{References}

[1] M. Barcau, Isogeny covariant differential modular forms and the space of elliptic curves up to isogeny, Compositio Math., 137 (2003), 237-273.

[2] A. Buium, Differential characters of Abelian varieties over p-adic fields, Invent. Math. 122 (1995), 309-340.

[3] A. Buium, Differential modular forms, J. reine angew. Math., 520 (2000), 95-167.

[4] A. Buium, A differential equation satisfied by the arithmetic Kodaira-Spencer class, preprint.

[5] C. Hurlburt, Isogeny covariant differential modular forms modulo p, Compositio Math., 128, (2001), 17-34.

[6] N. Katz, p-adic properties of modular schemes and modular forms, LNM 350, Springer Verlag 1973, 69-190.

[7] S. Lang, Introduction to Modular forms, Springer, 1976.

University of New Mexico, Albuquerque, NM 87131

E-mail address: buium@math.unm.edu 